\title{
EL MOVIMIENTO 'COCALERO' EN BOLIVIA DURANTE LOS AÑOS 80 Y 90: SUS CAUSAS Y SU SU DESARROLLO
}

\author{
Šárka Malá \\ Universidade Carlos/Praga - República Tcheca
}

Resumen: El presente articulo trata sobre el movimiento campesino de los productores de coca en Bolivia. En el presentamos un análisis sobre el nacimiento y el desarrollo del movimiento cocalero durante las décadas de los 80 's y 90's en el marco del contexto político y social boliviano de ese momento. Además trataremos de observar el estado actual del movimiento, una vez que el partido del cual es uno de los fundadores, llega al poder.

\section{The Cocalero Movement in Bolivia during the 80's and the 90's: its causes and development.}

\begin{abstract}
In the present paper we propose an analysis of the emergence and the development of the coca cultivating peasant's movement in Bolivia in the 1980 and 1990. We consider here the causes of the rise of the 'cocalero' movement in the wider politic and social context of the country in this historical moment. As well, we develop a reflexion about the development of the movement after that the political party MAS, who's it was one of the charter members, arrives in the government.
\end{abstract}

\section{Introducción}

El movimiento 'cocalero', en tanto organización de campesinos productores de la hoja de coca, nació en el contexto de un proceso de organización de los campesinos de los departamentos del Chapare y de los Yungas que se dió entre las décadas de los 80 y los 90. Ellos se perfilaron como movimiento social a partir de su oposición a las políticas de erradicación de la hoja de coca, las cuales fueron llevadas a cabo durante el mismo periodo. En cierto sentido, su estatus alcanzado como actor social fundamental de la política boliviana puede ser interpretado como una consecuencia directa de la importancia nacional e internacional que tomaron las políticas de erradicación de la coca.

En este articulo, nosotros intentaremos de mostrar el desarrollo de este movimiento durante los años 1980 y 1990, así como también las causas que le valieron su ascenso en la política nacional, llegando a constituirse como la primera fuerza de oposición. Al mismo tiempo, vamos a observar su evolución durante 
la segunda mitad de la década de los 90's, luego de la fundación del partido campesino Asamblea de la Soberanía de los Pueblos (ASP). Finalmente, vamos a dejar abiertas algunas interrogantes sobre el rol de este movimiento entre 2002 y 2005, apuntando a entender la pertinencia o no de seguirlos catalogando como un movimiento social.

\section{La producción de la coca en Bolivia: los Yungas y el Chapare}

En la Bolivia de las décadas de los 80's y 90's, la gran mayoría de la coca se produce fundamentalmente en dos regiones: la provincia Yungas, situada en el departamento de La Paz, y en la región llamada Chapare, la cual se encuentra en el departamento de Cochabamba ${ }^{1}$. La provincia de los Yungas, reconocida desde 1988 por la Ley $1008^{2}$ como una zona tradicional de producción de coca, es una región que está situada en la zona oriental de los Andes. La tradición de la producción de coca en los Yungas remonta muy lejos en la historia, antes de la Conquista, como lo demuestran diversos estudios arqueológicos. Aun cuando esta zona fue designada como una región para el cultivo legal de la coca, en los años 90's las plantaciones superiores a las 12.000 hectáreas fueron destinadas a la erradicación. Sin embargo, esta región no vivió con la misma intensidad 'la guerra contra la coca', como lo fueron otras zonas de producción de este cultivo.

La zona del Chapare se sitúa en una región tropical que, en el transcurso de la historia colonial y republicana, vivido diversas olas de inmigración. La colonización moderna, es decir, la que ha supuesto el establecimiento definitivo de sus pobladores, fue realizada en diversas etapas. Ella comenzó en los años 1920 con la construcción de la primera ruta hacia las tierras bajas del noreste del departamento de Cochabamba. Luego, en 1953, en el marco de la reforma agraria, las promesas gubernamentales atrajeron nuevos colonos. En los años 70's, el incremento de la demanda de la industria de la cocaína provoco un aumento importante de la producción de la coca en el Chapare y con ella se inició una nueva ola de inmigración de nuevos colonos que se instalarían en la región ${ }^{3}$. La producción se convirtió pronto en monocultivo debido al alto provecho económico que se podía obtener con las cuatro cosechas anuales que producen estos cultivos. La ultima ola de inmigración hacia el Chapare está ligada al "boom de la coca" que se produjo al comienzo de la década de los 80 's, cuando la región se convierte en una zona atractiva para muchos bolivianos, ya no exclusivamente campesinos. Por otra parte, además de los nuevos pobladores procedentes de las tierras pobres del altiplano, en su mayoría indígenas de las etnias quechua y aymaras, el Chapare acoge una inmigración masiva de mineros que habían sido despedidos antes o durante el cierre de las minas en $1985^{4}$. Un gran número de ellos fueron entonces relocalizados en las zonas de colonización, donde se encuentran con los campesinos 
que ya estaban instalados. Estos mineros traen consigo una tradición de lucha social que influenciara la organización sindical de los campesinos. Los despidos masivos y la crisis de la agricultura que provocó la reorganización económica neoliberal trajeron consigo la llegada de nuevos colonos a esta región ${ }^{5}$. En este momento, la economía que giraba en torno a la coca, se convirtió en una suerte de solución para el alza de los niveles de desempleo ${ }^{6}$.

\section{La breve historia de la lucha contra la coca.}

El regreso a la democracia en 1982 y el deseo de obtener la asistencia de los Estados Unidos para la reforma de la economía, obliga al Estado Boliviano a distanciarse de la producción de coca y de la cocaína. Llevados por la presión internacional en general y de los Estados Unidos en particular, el gobierno boliviano se compromete de manera mas importante en la lucha contra la droga. Durante el mandato de Hernán Siles Zuazo (1982-1985), se lleva a cabo, bajo el impulso norteamericano, un proyecto de erradicación de la coca cuyo destino era la industria del tráfico de drogas. De hecho, la asistencia de los Estados Unidos en la reforma económica estaba condicionada al compromiso boliviano con la lucha contra las drogas. Es así como en 1983 son creadas las Unidades Móviles de Patrullaje Rural (UMOPAR), las cuales eran unidades militares creadas con la finalidad de compensar la ausencia del Estado en ciertas áreas del territorio nacional.

Los objetivos que se propuso el Estado en materias de lucha contra la droga y la eliminación de los cultivos de coca, encontraron desde el comienzo une fuerte oposición por parte de los productores de coca. Estos organizaron las primeras acciones de protestas, generando así la apertura de negociaciones con el gobierno. Sin embargo, luego de la aplicación del programa de erradicación de la coca, el dialogo entre el gobierno y los productores estará marcado por un ciclo repetitivo, descrito por Roberto Laserna: acción - contracción - negociación - formulación de acuerdos - ruptura de los acuerdos? ${ }^{7}$. El gobierno, bajo presión norteamericana, se coloca en posición de fuerza frente a los cocaleros. El optara por la criminalización y la estigmatización del movimiento. Por su parte, los cocaleros utilizaron los métodos de movilización heredados del movimiento obrero y minero. De la publicación de demandas y reivindicaciones que el gobierno no tomó en cuenta, los cocaleros pasaron a acciones de presión directa como los piquetes de rutas, las huelgas de hambre, la búsqueda de apoyo en otras organizaciones sociales nacionales e internacionales y la movilización de la opinión pública.

Bajo la presidencia de Víctor Paz Estensoro se instrumentó el "Plan Trienal de Lucha Contra el Narcotráfico". Este plan fue presentado por Bolivia en 1987 durante la Convención de Viena de Estupefacientes bajo el nombre de "Plan 
Trienal Antinarcóticos del gobierno de Bolivia”. Este es recibido por la comunidad internacional como el primer paso institucional del gobierno boliviano para limitar el problema del tráfico de drogas, la producción de la coca y de la cocaína ${ }^{8}$. En este contexto, el gobierno de Paz Estensoro crea en 1987 las "Fuerzas Especiales de Lucha contra el Narcotráfico" (FELCN), la cual será una policía especializada en la lucha contra el trafico de drogas. La Ley que impulsa esta nueva política es promulgada el 18 de julio de 1988 y es conocida bajo el nombre de "Ley del régimen de la Coca y sustancias controladas" o simplemente "Ley 1008". Ella determina por la primera vez tres zonas de producción para la coca: la primera, de tipo tradicional y por consecuencia lícita, destinada al acullico, a usos medicinales o a rituales, los cuales no generan ningún tipo de dependencia (provincia de Yungas); la segunda, llamada "en transición", en la cual la producción de coca, fruto de un proceso de colonización espontánea, ha engendrado una expansión de cultivos excedentarios para dar respuesta a la demanda mundial de estupefacientes (la zona del chapare) y; una tercera, denominada ilícita, la cual está compuesta por el resto del territorio, donde la producción de coca está prohibida de forma inmediata y con carácter indefinido. La Ley provocara protestas y críticas radicales de los productores de coca y de ciertos sectores de la sociedad.

En el contexto del Plan Trienal, el gobierno de Jaime Paz Zamora (19891993 ) continúa desarrollando el programa de producción alternativa. Sin embargo, la erradicación continúa según las condiciones que fueron establecidas en la Ley 1008. En 1992 el gobierno boliviano firma la Declaración de Cartagena, el cual puede ser considerado como un acuerdo de militarización de la lucha antidroga ${ }^{9}$. Por el lado de los "cocaleros", la situación desencadenara nuevas movilizaciones que provocaran el reforzamiento interno del movimiento. En consecuencia, las tensiones, el ambiente de desconfianza general y la militarización de la región del Chapare ocurrida desde el final de los años 80 's, producirán una escalada y radicalización del conflicto.

El gobierno de G. Sánchez de Lozada (1993-1997) lleva a cabo en noviembre de 1994, nuevamente bajo presión del gobierno de Estados Unidos, el proyecto bautizado como "Opción Cero". Este nuevo programa supone la destrucción de todos los cultivos de coca en el Chapare y la transformación de la zona en Parque Nacional o en Zona Industrial. Este proyecto busca relocalizar los campesinos afectados hacia regiones donde recibirían nuevas tierras y una indemnización para el desarrollo de nuevos tipos de cultivos ${ }^{10}$. De esta forma, la zona se convierte en el epicentro de la lucha contra las drogas, generando su militarización y trayendo como consecuencia el nacimiento de un núcleo de oposición al programa constituido por los productores de coca.

Finalmente, durante el segundo mandato del General Hugo Banzer (19972001) y el de carácter interino de Jorge Quiroga (2001-2002) nuevas iniciativas 
son lanzadas para intentar controlar el problema de la producción excedentaria de la coca. En 1997 es llevada a cabo una nueva campaña de erradicación forzada, denominada "Plan Dignidad", la cual supuso una mayor militarización de la zona del Chapare.

En 2002, G. Sánchez de Lozada retorna el gobierno y pone como principal eje de su gobierno la privatización de los hidrocarburos y la continuación de los planes de lucha contra la producción de la coca. Luego de los eventos de la "guerra del Gas", Sánchez de Lozada renuncia al cargo, dejando de forma interina a Carlos Mesa como Presidente hasta el 2005.

Luego de su elección como presidente, el antiguo líder ‘cocalero’Evo Morales, expresa su voluntad de parar la política de erradicación forzada de la coca y de militarización de las zonas de cultivo. Durante su gobierno, se ha abierto el dialogo con el movimiento 'cocalero' sobre los temas de la erradicación y la sustitución de cultivos. Con su famosa consigna “¡Coca si, Cocaína no!” y con el programa "Cocaína cero", el gobierno mostrara su determinación de hacer una diferencia entre esos dos elementos. En este sentido, su gobierno creará el viceministerio de la coca, el cual se convertirá en el principal actor estatal responsable de la promoción del uso lícito de la hoja de coca y de la erradicación de cultivos excedentarios. A partir de esta institución la erradicación será reglamentada por un nuevo plan de compensación denominado "Control Social". Sin embargo, como veremos mas adelante, este nuevo programa también será la fuente de numerosos desacuerdos entre el gobierno y los productores de coca, así como también entre diferentes fracciones de los cocaleros mismos.

\section{El Nacimiento y el desarrollo del movimiento 'cocalero'}

Para comprender la especificidad y el desarrollo del movimiento 'cocalero', nos parece necesario ubicarnos en la década de 1970, momento en el cual empiezan a constituirse como organización social netamente diferenciada del campesinado. Como ya hemos señalado, hacia finales de los 70's, la dictadura de Hugo Banzer llevó a cabo un proyecto para la producción de coca a gran escala, así como también una campaña de colonización de las zonas tropicales. Los nuevos migrantes se encuentran con los antiguos productores de coca, llegados a la zona durante los años 70's, y comienzan a integrarse en los sindicatos que existían para ese momento. En efecto, tanto para el resto del campesinado como para los obreros, esos sindicatos representan una forma importante de organización social. En los años 80's, los sindicatos "cocaleros", adquirieron un poder mas grande que el que detentaban otros sindicatos campesinos. La gran importancia de estas organizaciones de base, puede ser explicada a partir de la ausencia del Estado en las zonas recientemente colonizadas. Es así como los sindicatos "cocaleros", manejan 
casi la totalidad de la vida social de las comunidades, implicándose en actividades que van desde la comercialización de la hoja de coca, hasta la educación de los niños o la organización de eventos deportivos y culturales ${ }^{11}$.

'En la zona del Chapare, el sindicato reagrupa una población muy heterogénea. Luego de la migración proveniente del altiplano boliviano, una gran parte de los colonos productores de coca son de origen quechua y aymara. De igual forma, la llegada de los mineros influenció de forma considerable muchos de los aspectos de las organizaciones de base. En este sentido, para muchos observadores, esta experiencia sindical previa, será un elemento importante para entender el éxito del movimiento 'cocalero'.

La proliferación masiva de sindicatos, los llevo a organizarse en diversas centrales y federaciones ${ }^{12}$. En 1982, con el apoyo de la COB, los 'cocaleros' crean la Confederación Nacional de Colonizadores de Bolivia (CNCB), organización independiente y afiliada directamente a la COB. De hecho, esta organización es una de las primeras que se emancipa de la tutela gubernamental que se dio durante el pacto militar-campesino ${ }^{13}$.

En los años 80's, luego del desarrollo del conflicto con el Estado, los sindicatos 'cocaleros' se convierte progresivamente en un actor muy influyente sobre la escena sindical nacional. A partir de la defensa de su producción, ellos se perfilan rápidamente como un movimiento de oposición con una capacidad de influencia importante. Según José A. Arrueta, su lucha contra el plan de erradicación de la coca les diferencia del resto de luchas del movimiento campesino, llegando a ser denominados por el propio Estado durante los años 80's como movimiento 'cocalero', para diferenciarlos de otras organizaciones sociales ${ }^{14}$.

Durante el inicio de la década de los 90's, la organización sindical 'cocalera' sigue desarrollándose. En el contexto del combate por la coca y con la finalidad de unificar las políticas de lucha contra su erradicación es fundada la Federación Especial del Trópico de Cochabamba - FETCTC, la cual pasará a tener el nombre de "Coordinadora" a partir del año 2000. Para este momento, la zona del Chapare ya cuenta con 30 centrales sindicales organizadas en 6 federaciones: Federación Yungas del Chapare (provincia del Chapare), Federación Trópico de Cochabamba (provincia del Chapare), Federación Centrales Unidas (provincia de Tiraque), Federación Chimore (provincia de Carrasco), Federación Carrasco Tropical (provincia de Carrasco) y Federación Mamore (zona Entre Ríos) ${ }^{15}$.

Aprovechando la importancia de su movimiento, los "cocaleros" se lanzan en la búsqueda de alianzas para influir en el seno de otras organizaciones sindicales. De igual forma, extienden su influencia en la esfera política y mediática, estableciendo relaciones con sectores de la Iglesia católica y con diversas ONG's nacionales e internacionales. 

su desarrollo

Durante la década de los 80 's los cocaleros ganaron influencia en el seno de organizaciones sindicales nacionales como la CSUTCB y la COB. Esto es comprensible, a partir de la constatación que las organizaciones cocaleras son las únicas que cobran una significación combativa en un contexto de reflujo de las luchas sindicales ocasionado por los programas de ajuste económico. Esto les valió convertirse en la vanguardia del movimiento sindical, remplazando al katarismo en el mando de las organizaciones campesinas como la CSUTCB en $1985^{16}$. Gracias a su creciente influencia política, los representantes cocaleros consiguen colocar la problemática de la producción y del consumo de la hoja de coca en el centro de la agenda política de la CSUTCB. Ya para 1987, se funda en el seno de esta organización la Comisión de la Coca, la cual se convertirá en una instancia permanente de dicha organización. De igual forma, esta problemática pasara a ser una fuente de atención de la comisión política y económica la CSUTCB. Al igual que en la CSUTCB, la influencia de los 'cocaleros' se incrementa en el seno de la COB. La combatividad creciente de los productores de coca, les permitió pedir una mayor representación en la COB por la vía de la CSUTCB. Aun cuando para la época los "cocaleros" disponen apenas de 4 representantes en la $\mathrm{COB}$, su influencia parece ser mayor a la que les corresponde por los puestos que ocupan. Rápidamente, ellos ganarán el respeto des los viejos militantes de la COB, sumándolos a la defensa de su causa. En este sentido, Kevin Healy menciona el ejemplo de Filemón Escobar, antiguo líder del movimiento sindical y portavoz de la $\mathrm{COB}$ durante muchos años, quien tuvo una participación muy activa en las negociaciones entre el gobierno y los "cocaleros". Otra personalidad importante del movimiento obrero, Juan Lechin, será contratado por los "cocaleros" como consultor profesional de las federaciones del Chapare ${ }^{17}$.

Durante este proceso de expansión del movimiento "cocalero", viejas organizaciones políticas en declive intentan aprovechar las posiciones alcanzadas por los productores de coca. Luego de la segunda mitad de los años 80's y del fracaso de la UDP ${ }^{18}$ de Siles Zuazo (1982 - 1985), los partidos políticos de izquierda se encuentran sumergidos en una crisis interna, la cual se manifiesta a través de sus frecuentes divisiones y la pérdida de su legitimidad. Es así como estos partidos intentan aliarse con un movimiento social que se encuentra en pleno ascenso. Los « cocaleros », con su creciente poder en el seno de las organizaciones sindicales nacionales y un discurso innovador, se convierten en un aliado ideal para estos partidos. Por su parte, el movimiento cocalero debe afrontar la creciente presión del gobierno y de las fuerzas armadas. Esto motivara su búsqueda de acceso al poder por la vía de alianzas con los partidos políticos de oposición: el "Movimiento Bolivia Libre" (MBL) y el "Partido Socialista" (PS); o con coaliciones de diversos partidos: el "Eje de Convergencia" 19 y la "Izquierda Unida"20. 
En este contexto y el de una creciente mediatización de la lucha "cocalera" contra el plan de erradicación, los partidos de izquierda adoptan la "causa de la coca" en sus programas, con el fin de asegurarse el voto del electorado campesino. Al igual que en el pasado en las zonas mineras, los partidos de oposición hicieron del Chapare una zona privilegiada en el reclutamiento de sus militantes y de votos electorales. Es así como entre 1985 y 1989 la "Izquierda Unida” y el MIR ganaron las elecciones en las provincias del Chapare, mientras que los partidos en el poder (MNR y ADN) tuvieron registros de retroceso significativo.

\section{Las causas de la ascensión de los 'cocaleros' al poder, el nacimiento del movi- miento social}

Hemos visto hasta aquí como en el transcurso de la década de los 80's y de la primera mitad de los 90's los sindicatos 'cocaleros' se convirtieron en un actor influyente de la escena sindical y política boliviana. Esta tendencia puede extrañarnos, si al mismo tiempo observamos el éxito que tuvo el gobierno para destruir los sindicatos mineros durante la década de los 80's, quienes representaron durante mucho tiempo la primera fuerza de oposición sindical del país y quienes contaban con una larga experiencia de lucha social. Los autores que trabajan este problema insisten frecuentemente sobre algunas condiciones claves que permiten explicar el crecimiento y la resistencia de los cocaleros ante los intentos de desmantelamiento por parte del Estado. Junto a Harry Sanabria ${ }^{21}$, vamos a tratar de entender cuales fueron esas causas.

Para comprender las causas del éxito de los cocaleros, hay que señalar que estos representaban sectores de la economía privada y que gozaban de una cierta autonomía con respecto al Estado. Las zonas de producción de coca se desarrollaron al margen del Estado, lo cual dificultaba su capacidad de control aun antes de la puesta en práctica de las políticas de erradicación de 1982. Es así, como a falta de medios de presión económica, el Estado encuentra una dificultad para subordinar a los sindicatos "cocaleros".

En segundo lugar, desde el punto de vista económico, a partir de los años 70's las ganancias de la producción de coca alcanzaron una representación importante dentro del presupuesto nacional. Como lo subrayan Roberto Laserna y otros autores, al momento de la aplicación de las reformas económicas, las cuales coincidieron con el gran "boom de la Coca", la importancia económica de esta actividad tuvo un crecimiento mayor ${ }^{22}$. Mientras la crisis económica golpeaba a los sectores privatizados, entre ellos la minería, la zona del Chapare tuvo una nueva ola de inmigración, que implicó el crecimiento en el numero de familias dedicadas a la producción de la coca. Paralelamente, los sectores altos de la sociedad boliviana (los mismos que poyaron las reformas neoliberales) tenían relaciones con las 
industrias de producción y de tráfico de la coca. La importancia de este fenómeno, puede ser ilustrado a partir del hecho que Bolivia se convirtió en ese momento en el segundo productor mundial de la $\operatorname{coca}^{23}$.

En tercer lugar, y directamente asociado con la ambigüedad del "problema de la coca", hay que señalar que tanto el Estado como las élites bolivianas no mostraron una determinación unificada sobre la erradicación de la coca, como si fue el caso en el cierre de las minas ${ }^{24}$. El sector privado, de forma directa o indirecta, obtuvo provecho de estos cultivos. En este sentido, podemos observar como en las disputas sobre la erradicación, los productores de coca obtuvieron el apoyo de la federación nacional de comercio. Además de esto, la estrategia escogida por el Estado de militarizar progresivamente las zonas en cuestión para erradicar la coca, siguiendo las recomendaciones de los Estados Unidos en la lucha contra las drogas, fueron estrategias que se mostraron poco acertadas para el contexto general que vivía el país durante la segunda mitad de la década de los 80 's. Esto permitió a los sindicatos "cocaleros" articular una doble estrategia. Por una parte, desarrollar una interpretación que presentaba la militarización del Chapare como una agresión contra los campesinos y por ende como una violencia excesiva por parte del Estado. Y por otra parte, la obediencia obsesiva del Estado a los consejos de Washington junto a la presencia de tropas norteamericanas en la zona del Chapare, les permitió a los "cocaleros" presentar estos hechos como un nuevo tipo de "colonialismo". Esto articulado con los programas de reforma económica, les permitió a los sindicatos capitalizar un sentimiento anti-norteamericano ya bastante extendido entre la población.

En cuarto lugar, cabe señalar la capacidad de los "cocaleros" para establecer alianzas con otras organizaciones sociales. Al ser el Chapare el lugar de encuentro de distintos sectores marginalizados de la sociedad boliviana, entre ellos antiguos sindicalistas mineros y campesinos de origen quechua y aymara, se juntaron distintas experiencias de lucha y organizativas, multiplicándose así los contactos con otras organizaciones sociales y generando una flexibilidad organizativa que les permitió reforzarse frente a los ataques del Estado ${ }^{25}$.

Inspirados por las experiencias de los sindicatos aliados, los "cocaleros" desarrollaron toda una gama de acciones de protestas que van desde el piquete de rutas estratégicas, hasta huelgas de hambre, pasando por marchas hacia la Capital. Esos eventos despertaron la atención de medios locales y nacionales, logrando imponer el tema de la coca como un problema central en el debate nacional. Esta magnitud alcanzada por los cocaleros, puede ser entendida también a partir del desarrollo de su discurso. El argumento de los 'cocaleros' señalaba el hecho que la hoja de coca era una planta sagrada de las poblaciones de origen quechua y aymara del altiplano, cuyo cultivo debía ser legitimo. Esto, acompañado al alto porcentaje 
de la población indígena en Bolivia, les permitía presentar la planta de la coca como una planta nacional sagrada, su consumo como una costumbre boliviana propia y su cultivo como parte integrante del patrimonio histórico. De esta manera, constatando el esfuerzo del Estado por erradicar esta planta y la ausencia de una voluntad real de coordinar este proceso con sus productores o por proponer un trabajo alternativo para estos, los "cocaleros" pasaron a acusar al gobierno de traidores del pueblo boliviano. Por otra parte, la presión ejercida por los Estados Unidos para erradicar la coca, le valió al gobierno la acusación, por parte de los productores, de ser servidores de la voluntad de Washington. Así, el Estado es acusado de haber vendido la soberanía nacional y la lucha de los 'cocaleros' pasa a ser una lucha por la recuperación de esta. Finalmente, cabe remarcar que estas interpretaciones discursivas encuentran resonancia con las de otro momento de la historia boliviana, cuando en la revolución de 1952, las elites del país fueron acusadas de ser aliadas de explotadores extranjeros, siguiendo una lógica neocolonial.

Con este discurso nace también la construcción de una identidad propia del movimiento. Se trata de la interpretación hecha por los propios 'cocaleros' sobre sí mismos, sobre su identidad y sobre su rol histórico en la lucha por la defensa de la producción de coca. Apoyados por sus múltiples alianzas, los 'cocaleros' se presentan como los portavoces del pueblo, de los sectores marginales de la sociedad boliviana. Además, identificándose como campesinos tradicionales e indígenas, los 'cocaleros' bolivianos encontraron un argumento de justificación étnica para la producción de la hoja de coca. Es en este sentido, que uno de los grandes temas del discurso de este movimiento se convirtió entonces (más allá de la defensa del cultivo de la coca) en la defensa de la identidad indígena ${ }^{26}$.

Podemos ver con curiosidad que la importancia de este acento sobre la etnicidad encuentra sus reflejos en la literatura de las ciencias humanas. Por ejemplo, el investigador boliviano, Xavier Albo, clasifica al movimiento 'cocalero', como un 'movimiento indianista' en su libro Pueblos indios en la politica ${ }^{27}$. El líder del movimiento, Evo Morales, es presentado como uno de los grandes personajes de origen indígena que lograron hacer avanzar la lucha reivindicativa del movimiento indígena. De la misma forma, la lucha por preservar los cultivos de coca es comprendida como parte integrante de las reivindicaciones indígenas de base. Este trabajo refleja la transferencia de la problemática de la erradicación de la coca al dominio de las reivindicaciones indígenas. Al mismo tiempo, revela la influencia del movimiento 'cocalero' en el movimiento indianista de ese momento.

En términos generales, podemos observar que durante las décadas de 1980 y 1990, gracias a diversos factores, los sindicatos se desarrollaron y ganaron una influencia importante en el seno de las organizaciones sindicales, así como 
también, en escenarios políticos y sociales más amplios que los meramente sindicales. Las demandas iniciales del movimiento cocalero comprendían algunos elementos de base: la ejecución de programas de desarrollos alternativos, la suspensión del uso de las fuerzas armadas en la erradicación de la coca, el respeto de los derechos humanos en las zonas de erradicación y el respeto de los acuerdos firmados. A estas demandas, fueron agregadas las de sus aliados, ensanchando así la base reivindicativa del movimiento ${ }^{28}$. Además de estas demandas, cada vez mas numerosas, hemos visto igualmente que en el transcurso de este periodo los "cocaleros" ganaron une identidad propia en tanto movimiento social ${ }^{29}$. Fuera de su carácter social, el movimiento adopta una dimensión étnica e indígena. Nosotros pensamos que es precisamente esta combinación del aspecto social y cultural lo que ha condicionado el éxito del movimiento durante la década siguiente.

\section{El movimiento 'cocalero' a partir de 1995: ¿un apoyo social del partido polí- tico MAS o un movimiento social autónomo?}

Las propias experiencias del movimiento 'cocalero' y las de sus alianzas políticas, así como también su oposición a los partidos tradicionales, empujaron a la creación de un partido político propio que articulase al movimiento 'cocalero' con otros sectores del movimiento campesino. En 1992, durante la Asamblea de la CSUTCB y en ocasión de la conmemoración de la invasión española en el continente americano, se abrieron las discusiones sobre la creación de un instrumento político del campesinado ${ }^{30}$. La idea, cuyo uno de sus principales impulsores fue Evo Morales, fue retomada durante la asamblea que se dio entre el 25 y el 27 de marzo de 1995, creándose así el partido "Asamblea de la Soberanía de los Pueblos" (ASP). Aprovechando el proceso de descentralización municipal que había sido puesto en marcha durante ese mismo año, el nuevo partido se presento a las elecciones municipales de diciembre de 1995 bajo el registro de la "Izquierda Unida" (IU), aliado principal de los 'cocaleros' de Cochabamba ${ }^{31}$. El resultado es significativo: la ASP-IU gana las elecciones en el departamento de Cochabamba, obteniendo 10 alcaldías, 49 consejeros municipales y 6 consejeros departamentales. Además, de esto, también obtendrán otros 5 puestos de consejeros en los departamentos del altiplano ${ }^{32}$.

Este evento, puede ser considerado como el inicio de una nueva fase del movimiento 'cocalero'. Por una parte, podemos observar una nueva etapa en su proceso de expansión política ${ }^{33}$. Por otra parte, podemos interpretar este momento como el comienzo del desarrollo de un movimiento político independiente, fruto de las alianzas con otros sectores sociales y partidos de izquierda. Desde ese punto de vista, el movimiento 'cocalero' continuara su curso durante los años 90's al lado de este movimiento político, el cual absorberá nuevos grupos y nuevos 
temas reivindicativos, ensanchando así su perfil como un partido de izquierda. Los análisis sobre este partido político posteriores al año 2000, solo dan al movimiento 'cocalero' un estatus fundador, mientras que los aspectos étnicos cobran una mayor relevancia. De esta forma, en el discurso de la ASP (y más tarde en el del MAS) la causa de la coca se diluye en otras reivindicaciones que cobran mayor importancia. El problema de la producción de la coca estará ligado a la defensa del consumo tradicional, la insistencia de su importancia como valor cultural y con el discurso antiimperialista ${ }^{34}$.

Desde este punto de vista, podemos también comprender la crisis interna que vive el movimiento campesino en 1998. Este año, la ASP se divide en dos ramas y presentan dos listas de candidatos para las elecciones municipales de 1999. La rama dirigida por Alejo Veliz, la cual guardará el nombre de la "Asamblea de la Soberanía de los Pueblos", ganará 28 puestos en los consejos municipales y 5 alcaldías en Cochabamba. La rama dirigida por Evo Morales adoptará un nuevo nombre: "Instrumento Político para la Soberanía de los Pueblos" (IPSP) y se presentará a las elecciones bajo el registro del MAS-U ${ }^{35}$. Gracias a la popularidad de su líder, este partido obtiene un mejor resultado que el de la ASP, ganando 79 puestos en los consejos municipales en 7 departamentos ${ }^{36}$, mayoritariamente en aquellos de producción de la coca y en las regiones andinas.

Sin embargo, la gran victoria del MAS llegará en las elecciones de 2002 y de 2005. En 2002 el partido obtiene el 22,94\% de los votos a nivel nacional, terminando segundo, dos puntos por debajo del partido ganador, el MNR. Asegurándose 8 senadores y 36 diputados al parlamento, el MAS obtiene una victoria histórica sin precedentes para un partido indígena y campesino ${ }^{37}$. El resultado de las elecciones de 2005 sobrepasara aun más el éxito ya alcanzado en 2002. El antiguo líder 'cocalero' de origen aymara, Evo Morales, se convierte en el Presidente de la Republica y el MAS pasa a ocupar el primer lugar en el sistema político boliviano. Nuevas iniciativas de orden social son llevadas a cabo durante su gobierno, entre ellas la revisión de las políticas de erradicación de los cultivos de coca en coordinación con el propio movimiento 'cocalero'.

Al mismo tiempo, durante el periodo 2002-2005 el MAS ha insistido sobre la importancia dada a sus bases y al proceso de consulta con las mismas. Algunos autores ${ }^{38}$ hablan del carácter extra-sistémico de ese partido, quien esta siempre listo para poner en cuestión su estatus político, en provecho del regreso a sus orígenes, es decir, a las organizaciones sociales. A pesar de su éxito electoral, el MAS continúa asumiéndose como al margen del sistema político, cultivando sus nexos con los movimientos de base. Es así, como el movimiento 'cocalero', se encuentra entre las organizaciones sindicales nacionales, que sirven de base de apoyo al gobierno. 

su desarrollo

Sin embargo, luego de 2005, la imagen del MAS como movimiento social es cada vez más difícil de sostener. Aun cuando el intenta de perpetuar las practicas de dialogo con el movimiento sindical, el debe afrontar el proceso de institucionalización que conlleva su nueva posición política. Los sindicatos 'cocaleros' perpetúan su alianza con el gobierno de la misma forma que otros sindicatos campesinos, pero al mismo tiempo están a la espera de la realización de las promesas electorales concernientes al tema de la coca. El trabajo hecho hasta el momento por el gobierno, prevee revisar la validez de la Ley 1008 en algunas regiones ${ }^{39}$, mientras que en otras, la erradicación esta planteada en los mismos términos de la Ley de 1988, acompañada por un sistema de compensaciones a las comunidades en cuestión. Frente a estas políticas y sus insuficiencias, los productores no tienen la misma unidad que disponían en las décadas de 1980 o 1990, oscilando así entre la oposición y la cooperación con el gobierno. ${ }^{40}$

Así, luego de haber trazado el desarrollo del movimiento 'cocalero', podemos preguntarnos sobre su estado actual en la Bolivia de Evo Morales. ¿Son ellos todavía un movimiento social? ¿O se han diluido en diversas organizaciones que se desarrollan en la esfera política y social? ¿Ellos han perdido su importancia retornando a su condición inicial de movimientos a escala local? Aun si esas preguntas van mas allá de los objetivos de nuestro trabajo, basado en el análisis del desarrollo del movimiento, ellas abren nuevas perspectivas para comprender la actualidad boliviana. De igual forma, podríamos preguntarnos sobre el destino del resto de movimientos sociales que inicialmente le dieron vida al Movimiento al Socialismo.

\section{BIBLIOGRAFÍA}

ALBO, X. Pueblos indios en la política, La Paz: CIPCA, 2002.

ALENDA, S."Bolivie : les limites de la consolidation institutionnelle : l'érosion du pacte démocratique" in: Problèmes d'Amérique latine, No. 49, Paris, été 2003. ARRUETA R., J. A. Campesinado, coca y estado, Cusco: Centro de Estudios Regionales Andinos Bartolomé de Las Casas, 1994.

CASEN, C. "Système de partis et partis antisystèmes en Bolivie. Emergence du MIP et du MAS sur la scène politique bolivienne depuis 2000" in : Cahiers des Amériques Latines, $\mathrm{N}^{\circ} 46,2004$.

CASTILLO GALLARDO, M. "Movimiento cocalero en Bolivia. Violencia, discurso y hegemonía" in: Gazeta de Antropología, n²0, Santiago de Chile 2004, p. $20-35$. 
FARRO, A. L. Les mouvements sociaux, Montréal, Presse de 1'Université de Montréal, 2000.

FRANQUEVILLE, A. La Bolivie, d'un pillage à l'autre, Paris : IRD éditions, 2000 .

HEALY, K. "Coca, the State and the Peasantry in Bolivia, 1922 - 1988"in: Journal of Interamerican Studies and World Affaires, $\mathrm{n}^{\circ} 2 / 3$, Special Issue: Assessing the Americans War on Drugs, JSTOR, 1988.

HEALY, K. "Political Ascent of Bolivia's Peasant Coca Leaf Producers" in: Journal of Interamerican Studies and World Affaires, $\mathrm{n}^{\circ}$ 1, JSTOR, spring 1991.

LASERNA, R. "Bolivie : l'économie de la drogue » in : Problèmes d'Amérique latine, $\mathrm{N}^{\circ} 18$, Paris, juillet. - sept. 1995

LASERNA, R., CAMACHO N., CORDOVA, E. Empujando la concertación. Marchas campesinas, opinión publica y coca, La Paz: PIEB, 1999.

LEE VAN COTT, D. "From Exclusion to Inclusion: Bolivia's 2002 Elections" in: Journal of Latin American Studies, Cambridge University Press, N 35, 2003.

LINERA, Á. G. "El evismo: lo nacional-popular en acción”, OSAL, CLACSO, No. 19, julio 2006.

MANERO, E. A., SALAS, E. Mondialisation et "Nationalisme des Indes", Toulouse, Collection Méridiennes, 2007.

MAYORGA, F. "Bolivie: la gauche rurale et indigène au gouvernement", in : Problèmes d'Amérique latine, $\mathrm{N}^{\circ} 59$, Paris, hiver 2005 - 2006.

OBSERVATIOIRE GEOPOLITIQUE DES DROGUES, Nouveau désordre mondial (Rapport 1992 - 1993), Paris, Hachette, 1993.

OBSERVATIOIRE GEOPOLITIQUE DES DROGUES, Géopolitique des drogues - 1995 (Rapport annuel de l'OGD), Paris, La Découverte, 1995, 324 p.

PINTO OCAMPO, M., T., "Entre la represión y la concertación: Los campesinos en el Chapare y en el Putumayo", Buenos Aires: CLACSO, 2004.

QUIJANO, A. “Estado-nación y 'movimientos indígenas' en la región Andina: cuestiones abiertas", OSAL, N¹9, Buenos Aires: CLACSO, 2006.

RIVERA, P. A., AGUILO, F., LASERNA, R. El Chaparé actual: sindicatos y $O N G$ 's en la región, Cochabamba, Instituto latinoamericano de Investigaciones Sociales (ILDIS), 1990.

SANABRIA, H. "Consolidating Status, Restructuring Economies, and Confronting Workers and Peasants: The Antinomies of Bolivian Neoliberalism" in Comparative Studies in Society and History, Vol. 14, No. 3, Jul. 1999. 
su desarrollo

SPEDDING P., A. Kawsachun coca: economía campesina en los Yungas y el Chapare, La Paz: Fundación PIEB, 2004.

STEFANONI, P. "MAS - IPSP: la emergencia del nacionalismo plebeyo", OSAL, ${ }^{\circ} 12$, septembre - décembre 2003.

STEFANONI, P. "El nacionalismo indígena en el poder", $O S A L, \mathrm{n}^{\circ} 19$, juillet 2006.

TORRICO, T. M. “¿Que ocurrió realmente en Bolivia?”, Perfiles Latinoamericanos, FLACSO, $\mathrm{n}^{\circ}$ 28, juillet - décembre 2006.

\section{NOTAS}

I «El Chapare » es una denominación extendida que se aplica para designar tres provincias: el propio Chapare, Carrasco y Tiraque. La Capital económica y social de toda la zona es Villa Tunari, aun cuando esta ultima, lo es únicamente de la Provincia del Chapare. SPEDDING P., Alison, Kawsachun coca: economía campesina en los Yungas y el Chapare, La Paz, Fundación PIEB, 2004, p. 91.

2 La Ley 1008 o Ley del Régimen de la Coca y Sustancias Controladas fue adoptada el 19 de julio de 1988 como una respuesta del gobierno boliviano a las presiones internacionales de las que fueron objeto en el contexto de la lucha contra la producción de cocaína (y de la coca). Ella reconocía dos tipos de cultivos: la producción para el consumo licito de tipo tradicional (acallicu, infusiones, etc.) y la producción para la fabricación de la cocaína, la cual era ilícita. La Ley precisa cual era la cantidad que seria considerada como legal: 12000 hectáreas cultivadas en la zona de Yungas. El resto de la producción haría parte de los "cultivos excedentarios e ilícitos" y que por ende, estaría condenada a su eliminación.

3 Esta inmigración hacia las zonas tropicales fue incitada por el gobierno del general Hugo Banzer y fue conocida bajo el nombre de "la marcha hacia el oriente".

4 En total 85.000 mineros fueron despedidos en 1985. FRANQUEVILLE, A., La Bolivie, d'un pillage à l'autre, Paris, IRD éditions, 2000, p. 21.

5 Según algunos autores, además del crecimiento de las tasas de desempleo, otros elementos como la explosión demográfica, incrementaron el numero de personas en edad de trabajar, lo cual jugó un papel importante en el desplazamiento de estos nuevos colonos a la región del Chapare. Ver: LASERNA, R, « Bolivie: l'économie de la drogue », Problèmes d'Amérique latine, No. 18, juillet - septembre 1995, p. $83-99$.

6 Como consecuencia de estos procesos migratorios, la población del Chapare se vio incrementada 10 veces entre 1967 y 1987. Así el Chapare paso de tener 24.000 habitantes en 1967 a tener aproximadamente 250.000 habitantes en 1987. Ibídem.

7 LASERNA, Roberto, CAMACHO B., Nathalie, CORDOVA, Eduardo, Empujando la concertación. Marchas campesinas, opinión publica y coca, La Paz, PIEB, 1999.

8 ARRUETA RODRIGUEZ, J. A., Campesinado, coca y estado, Cusco, Centro de Estudios Regionales Andinos Bartolomé de Las Casas, 1994.

9 OBSERVATIOIRE GEOPOLITIQUE DES DROGUES, Nouveau désordre mondial (Rapport 1992 1993), Paris, Hachette, 1993, p. 262.

I 0 OBSERVATIOIRE GEOPOLITIQUE DES DROGUES, Géopolitique des drogues - 1995 (Rapport annuel de l'OGD), Paris, La Découverte, 1995, pp. 198-199

I I En su artículo, Kevin Healy menciona un ejemplo poco conocido, como lo fue una reforma agraria local 
organizada por los sindicatos « cocaleros » en 1983. Esta iniciativa fue llevada a cabo bajo la protección de la coalición de la UDP y con la asistencia del Instituto Nacional para la Colonización. Esta reforma consistía, de la misma forma que la reforma de 1953, en la redistribución de tierras no utilizadas por los grandes propietarios. HEALY, K., « Political Ascent of Bolivia's Peasant Coca Leaf Producers », Journal of Interamerican Studies and World Affaires, $n^{\circ}$ I, JSTOR, spring 1991, p. 87 - 121.

12 Según María Teresa Pinto Ocampo, un sindicato de base cuenta con entre 10 y 300 personas. Una central agrupa entre 5 y 18 sindicatos y una federación reúne a varias centrales. PINTO OCAMPO, M., T., «Entre la represión y la concertación: Los campesinos en el Chapare », Buenos Aires, CLACSO, 2004, p. 5.

13 ALBO, X., "El sector campesino-indígena, actor social clave", Opiniones y Análisis, Fundemos, No. 52, La Paz, noviembre 2000, p. 92. Cité par: ALENDA, S., (2003), Op. cit., p. 88.

I 4 ARRUETA R., J. A., Op. cit., p.7

I 5 SPEDDING P., A., Op. cit., p.297.

16 HEALY, Kevin, ( 1991 ), Op. cit., p.93.

17 Ibídem.

18 "Unidad Democrática Popular".

19 Pequeña coalición de partidos políticos de izquierda: partido comunista, el Bloque Popular Patriótico, la Alianza Patriótica y el "MIR - Masis". HEALY, Kevin, ( 991 ), Op.cit., p. 105.

20 La Izquierda Unida es fundada en 1988 a partir de la unión del MBL, el Partido Comunista y el "MIR - Masis". Ibídem.

2 I SANABRIA, H, "Consolidating Status, Restructuring Economies, and Confronting Workers and Peasants: The Antinomies of Bolivian Neoliberalism", Comparative Studies in Society and History, Vol. 14, No. 3, Jul. 1999, p. 535 - 562.

22 LASERNA, R., "Bolivie : l'économie de la drogue" Problèmes d'Amérique latine, N I8, juillet. - sept. 1995, p. 83 - 99.

23 Kevin Healy en su articulo señala: "It is clear that, during the 1980's, the Chapare federations have become a major force within Bolivia's national political system. (...) This newly-acquired power derives from their influence over a major political issue which affects the drug-consuming industrial nations of the North. (...) The high priority, both national and international, accorded this focus on drugs has been a major factor in encouraging the growers to acquire the sophisticated political skills needed to defend their interests and interact with other interest groups in the Bolivian democracy". HEALY, K., ( 1991), Op.cit., p. I I I.

24 Para un estudio comparativo del movimiento minero y cocalero ver: SANABRIA, H., Op.cit. 25 Ibidem., p. 553.

26 Xavier Albo llama la atención sobre las relaciones reciprocas entre el desarrollo del discurso "cocalero" y el ensanchamiento de sus alianzas. Podríamos así desarrollar una reflexión sobre la doble formación de este discurso. A lo interno del movimiento sindical para ampliar y reforzar sus alianzas y; a lo externo, para tomar ventaja en su conflicto con el Estado. Así, sobre la coloración étnica tomada por el discurso "cocalero" Xavier Albo señala: "Esto permitió a los cocaleros en el Chapare estar 'más cerca' de un amplio sector boliviano que se identifica con la identidad étnica, permitiéndoles construir alianzas estratégicas que, a la larga, han sido indispensables para el posicionamiento político de sus demandas". ALBO, X., Pueblos indios en la política, La Paz, CIPCA, 2002, p.26.

27 ALBO, X, Op.cit.

28 Este fenómeno ha sido estudiado por Roberto Laserna. LASERNA, R, CAMACHO B., N. et CORDOVA E., E., Op. cit.

29 El concepto de Movimiento Social es muy amplio. El puede tener múltiples interpretaciones y definiciones según la corriente sociológica que intente interpretarlo. Sin embargo, diversos autores están de acuerdo sobre las siguientes características de los movimientos sociales: una red informal o extra-institucional de individuos movilizados, en oposición o conflicto con la 
red institucional por razones de recursos o valores de diverso tipo. Al interior del movimiento se desarrolla una solidaridad importante entre sus miembros, generándose así una identidad. FARRO, A. L., Les mouvements sociaux, Montréal, Presse de I'Université de Montréal, 2000. 30 LEE VAN COTT, D., "From Exclusion to Inclusion: Bolivia's 2002 Elections", Journal of Latin American Studies, No 35, 2003, Cambridge University Press, p. 763.

3I ALENDA, S., (2003), Op. cit., p.91.

32 LEE VAN COTT, D., Op. cit.

33 LINERA, Á. G., "El evismo: lo nacional-popular en acción”, OSAL, CLACSO, No. 19, julio 2006, p. $25-32$.

34 QUIJANO, A., "Estado-nación y 'movimientos indígenas' en la región Andina: cuestiones abiertas", OSAL, N¹9, CLACSO, Buenos Aires, juillet 2006, p. 59.

35 A partir de 1999 el IPSP se presenta con el registro del "Movimiento al Socialismo" (MAS), el cual era una vieja escisión de la Falange Socialista de Bolivia. ALENDA, Stéphanie, Op. cit.

36 Bolivia esta dividida administrativamente en 9 departamentos.

37 Esto suscitara numerosos análisis de observadores bolivianos y extranjeros, quienes buscaban las causas de ese resultado político inesperado. Entre los factores del éxito del MAS encontramos los factores mencionados anteriormente: la alianza con el movimiento indianista, la instrumentalización del sentimiento anti-norteamericano, el potencial político generado en el transcurso de las movilizaciones cocaleras, el descontento de la población con la crisis económica del país, la radicalización de las demandas étnicoculturales, la madurez política de los dirigentes experimentados por su trabajo sindical, la capacidad de innovar su discurso con el transcurso de los años y finalmente las reformas del proceso electoral que permitieron a los pequeños partidos participar de las elecciones municipales..

38 CASEN, C., "Système de partis et partis antisystèmes en Bolivie. Emergence du MIP et du MAS sur la scène politique bolivienne depuis 2000", Cahiers des Amériques Latines, N²6, 2004, p. 63 - 72.

39 Por ejemplo, en la región La Asunta. "Un pacto omite la 1008 y legaliza la coca en La Asunta”, La Razón, http://www.la-razon.com/versiones/20080919_006400/nota_256_67385I.htm.

40 "Cocaleros toman viceministerio de la coca", Bolivia Opina, 4 abril 2007, http://www.boliviaopina. com/showthread.php? $p=496$. 